Yervant Terzian, Daniel Weedman, Edward Khachikian, eds.

\title{
Flare in the Hydrogen Line Region of the NGC 3227 Nucleus on January 12-15, 1997
}

\author{
I.Pronik $^{1}$, L.Metik ${ }^{1}$, N.Merkulova ${ }^{1}$ \\ Crimean Astrophysical Observatory, Crimea, Ukraine
}

Introduction. The nucleus of NGC 3227 was classified as a Sy2 type before 1974, and a Sy1 type after 1974. The Seyfert type of the nucleus changed corresponding to its brightness: U brightness of the nucleus increased in 1974: $\Delta \mathrm{U} \sim 1^{m}$. Maximum brightness of the nucleus was observed in $1975-1977$.

Observations. 57 spectrograms in the spectral region $3700-7300 \AA \AA$ were obtained with the 6-m telescope on January 12-15, 1977 during maximum of the nucleus brightness. Spectral resolution was $\sim 8 \AA$. Seeing was $(1-3)^{\prime \prime}$.

Equivalent widths $\left(\mathrm{W}_{\lambda}\right)$ and profiles of the emission lines $\mathrm{H}_{\delta}, \mathrm{H}_{\gamma}, \mathrm{H}_{\beta}$, [OIII] $\lambda \lambda 4959,5007 \AA \AA, \mathrm{H}_{\alpha}+[\mathrm{NII}]$, and [SII] $\lambda \lambda 6717,6731 \AA \AA$ were averaged by series. Duration of the series of observation was $\sim 25 \mathrm{~min}$.
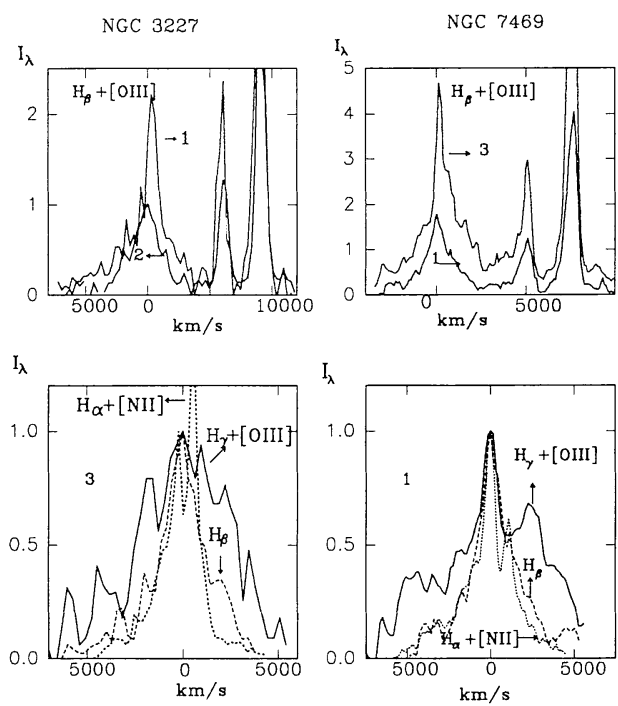

Figure 1. Night-to-night variations in line profiles (see text).

Equivalent widths of lines showed asynchronous variations. This fact leads us to suspect, that there were night-to-night variations of intensities of emission lines with upper limit $\sim 1.5$ times.

Profiles of Balmer lines $\mathrm{H}_{\gamma}, \mathrm{H}_{\beta}$ and $\mathrm{H}_{\alpha}$ in the spectrum of the NGC 3227 nucleus showed remarkable similarity in characteristics of night-to-night varia-

\footnotetext{
${ }^{1}$ Isaac Newton Institute of Chile, Crimean Branch
} 
tions with the same lines in the spectrum of the NGC 7469 nucleus (Pronik et al., 1997).

Comparison of results can be seen in the Figure, where figures on plots signify the ordinal numbers of nights from the beginning of the event. Profiles on the top panel, where $\mathrm{I}_{\lambda}$ are given in continuum units show that:

1. Central narrow peaks of the $H_{\beta}$ line are double in both cases.

2. The ratio $\mathrm{I}_{b} / \mathrm{I}_{r}$ of the narrow $\mathrm{H}_{\beta}$ line smoothly decreases from $\mathrm{I}_{b} / \mathrm{I}_{r} \geq 1$ to $\mathrm{I}_{b} / \mathrm{I}_{r} \leq 1$ when $\mathrm{W}_{\beta}$ is increased (i.e. continuum of the nucleus is decreased).

On bottom panel $I_{\lambda}$ of profiles are given in units of peak brightness of Balmer lines. These profiles show that:

3. Strong brightening of $\mathrm{H}_{\gamma}$ broad wings were observed with lag 1-2 days from the beginning of the brightening of the blue narrow $\mathrm{H}_{\beta}$ component.

Discussion. We suppose that night-to-night variability of profiles of Balmer lines in spectra of NGC 3227 and NGC 7469 nuclei are connected with short time flares in emission line regions of these nuclei. Two variable components of a narrow $\mathrm{H}_{\beta}$ line can reflect the existence of two variable streams in both nuclei. In that case, radial velocities of the streams emitting $\mathrm{H}_{\beta}$ light are about -250 $\mathrm{km} / \mathrm{s}$ and $-550 \mathrm{~km} / \mathrm{s}$ compare to the recession velocities for the galaxies NGC 7469 and NGC 3227 correspondingly.

The observational indication of the beginning of a short-time flare appears to be a relative brightening of the blue narrow peak of the $\mathrm{H}_{\beta}$ line and an increasing of continuum flux of the nucleus. Variations in streams approaching observers can be interpreted as an ejection from the nucleus with radial velocities of about $200-500 \mathrm{~km} / \mathrm{s}$. This ejection influences the gaseous regions emitting broad Balmer lines: brightening of narrow blue $\mathrm{H}_{\beta}$ component was accompanied by an increase with 1 day lag of the intensity of the broad $\mathrm{H}_{\gamma}$ wings with the radial velocities equal to $\pm 6500 \mathrm{~km} / \mathrm{s}$. Such high intensity of broad wings was not observed in the $\mathrm{H}_{\beta}$ and the $\mathrm{H}_{\alpha}$ profiles. One can argue that the emission of broad $\mathrm{H}_{\gamma}$ wings has an inverse Balmer decrement. Comparison with theoretical models permits us to suppose that this gas could be opaque, hot and inhomogeneous in physical conditions of plasma $\left(\mathrm{T}_{e}=25000 \mathrm{~K}, \mathrm{n}_{e}=10^{12}-10^{14} \mathrm{~cm}^{-3}\right)$, and it is ionized and excited mainly by a collisional process.

Almost simultaneous variability of the broad $\mathrm{H}_{\gamma}$ line and the narrow component of the $\mathrm{H}_{\beta}$ line during the flare shows that regions emitting broad lines and narrow lines are overlapped. These regions are not more than several light days $\left(\sim 4.5 \cdot 10^{15} \mathrm{~cm}\right)$ in dimension. One can speculate that regions of flares are excited by shocks acting inside jets.

The flare in NGC 7469 was observed during the minimum brightness of its nucleus (Pronik et al., 1997). The flare in NGC 3227 was observed during the maximum brightness of its nucleus. Therefore short time flares did not connect with the general brightness of nuclei of galaxies. One can suspect that there are two independent sources of nuclear activity. One of them is connected with the general brightness of the nucleus, and another one is not.

\section{References}

Pronik, I., Metik, L., \& Merkulova, N. 1997, A\&A, 318, 721 